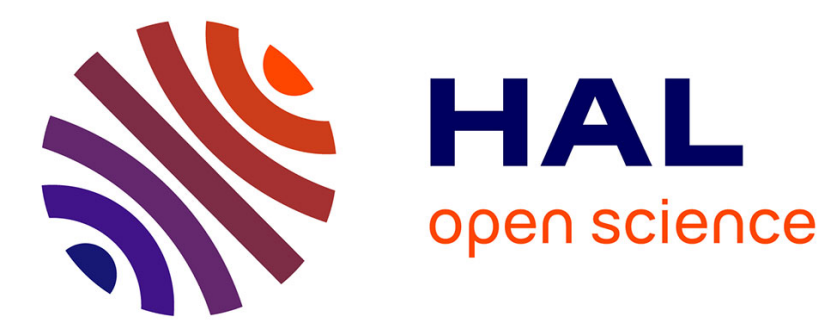

\title{
Catilina legatus. Considerazioni su un discusso frammento sallustiano
}

\author{
Gianpaolo Urso
}

\section{To cite this version:}

Gianpaolo Urso. Catilina legatus. Considerazioni su un discusso frammento sallustiano. KTÈMA Civilisations de l'Orient, de la Grèce et de Rome antiques, 2019, La rhétorique de la diplomatie en Grèce ancienne, 44, pp.197-207. halshs-02444372

\section{HAL Id: halshs-02444372 https://shs.hal.science/halshs-02444372}

Submitted on 17 Jan 2020

HAL is a multi-disciplinary open access archive for the deposit and dissemination of scientific research documents, whether they are published or not. The documents may come from teaching and research institutions in France or abroad, or from public or private research centers.
L'archive ouverte pluridisciplinaire HAL, est destinée au dépôt et à la diffusion de documents scientifiques de niveau recherche, publiés ou non, émanant des établissements d'enseignement et de recherche français ou étrangers, des laboratoires publics ou privés. 

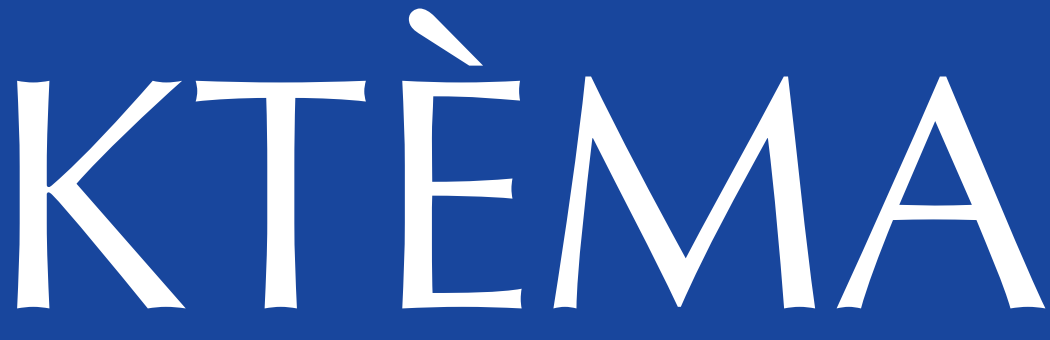

\section{CIVILISATIONS DE L'ORIENT, DE LA GRÈCE ET DE ROME ANTIQUES}

\section{La rhétorique de la diplomatie en Grèce ancienne}

Cinzia BeARzot, Laura Loddo

Laura LoDDo

Cinzia BeArzot

Paolo A. Tuci

Francesca Gazzano

Nicholas D. Cross

Davide Amendola

Jonathan R. W. PraG

Lorenzo CAMPAGNA

Cristina SORACI

Edith Foster

Walter LAPINI

Sylvain PERROT

Gianpaolo Urso

Anne JACQuemin
Political Exiles and Their Use of Diplomacy in Classical Greece

À propos du parallélisme entre deux discours d'ambassade à Sparte (Xén. Hell. V, 2, 12-19 et VI, 1, 4-16). 23

The Speeches of Theban Ambassadors in Greek Literature (404-362 B.C.)................ 33

Greek Ambassadors and the Rhetoric of Supplication. Some Notes ............................ 53

The (Im)balance of Power. Demosthenes' Complex Case

for an Alliance with the Megalopolitans.....

Presbeutikoi and Enteuktikoi Logoi in Hellenistic Interstate Relations.

Some Further Thoughts from an Epigraphical Perspective (c. 306-205 B.C.). 87

\section{Sicile hellénistique et romaine}

I.Sicily, Open Scholarship, and the Epigraphic Landscape of Hellenistic/Roman Sicily.

Trasformazioni urbanistiche in Sicilia alle origini della Provincia.

Riflessioni sul ruolo di Roma.....

Cultes et politique dans la Sicile du i ${ }^{\text {er }}$ siècle av. J.-C.

Le cas de la Vénus Érycine et de la Cérès d'Henna

\section{Varia}

Athens' Political Failures in the Central Chapters of Book 4 of Thucydides 163

Note interpretative e testuali alla col. XXII del Papiro di Derveni

La place de la musique dans la politique culturelle de Téos

dans la première moitié du $\mathrm{II}^{\mathrm{e}}$ siècle avant notre ère

Catilina legatus. Considerazioni su un discusso frammento sallustiano.

La dédicace aux Muses d'Eurydice fille de Sirrhas

(Plutarque, Sur l'éducation des enfants, 20) 


\section{Catilina legatus \\ Considerazioni su un discusso frammento sallustiano}

RÉsumé-. Un fragment des Histoires de Salluste évoque un siège mené par L. Catilina legatus. Cet épisode a été attribué pendant longtemps à la guerre civile de 83-82 ou aux opérations en Italie qui s'ensuivirent. Cet article vise à réexaminer le problème, en tenant compte du contenu du premier livre des Histoires et de certains faux présupposés qui caractérisent plusieurs reconstructions modernes. Il propose d'identifier le contexte le plus probable de ce siège avec le tumultus Lepidi de 78-77. Si cette hypothèse est plausible, Catilina aurait été legatus de Catulus ou de Pompée, Sullanae dominationis duces atque signiferi.

Mots-CLÉs-. Catilina, Salluste, Q. Lutatius Catulus (cos. 78), Pompée, Sylla

Aвstract-. A fragment of Sallust's Histories mentions a siege led by L. Catilina legatus. The episode has long been ascribed to the civil war of $83-82$, or to the subsequent operations in Italy. This paper aims to reassess the problem. It begins by considering the content of the first book of the Histories and some false assumptions which undermine most modern reconstructions, then argues that the most probable context of the siege is the tumultus Lepidi of 78-77. If so, Catiline would have been legatus of Catulus or Pompey, Sullanae dominationis duces atque signiferi.

Keywords-. Catiline, Sallust, Q. Lutatius Catulus (cos. 78), Pompey, Sulla

Le testimonianze antiche sulla giovinezza di Catilina, non molto numerose, sono centrate quasi esclusivamente sulla sua militanza tra i partigiani di Silla e sui presunti "crimini" che egli avrebbe commesso alla fine dell'82, durante le proscrizioni: l'uccisione del pretore M. Mario Gratidiano (Q. Cic., pet., 10; Cic. in Asc., tog. cand., p. 87, 90 Clark; Asc., ibid., p. 84, 87, 90 Clark; Sen., dial., 5.18.1-2; Plut., Sull., 32.4; scholia Bernensia ad Lucanum, 2.173), quella del cavaliere Q. Cecilio, suo suocero (Q. Cic., pet., 9), e quella di suo fratello (Plut., Cic., 10.3; Sull., 32.3) ${ }^{1}$. Una breve notizia di Sallustio si differenzia però dalle altre, perché non fa menzione di Silla e si riferisce a un contesto più propriamente bellico. Si tratta di un breve frammento del primo libro delle Historiae (fr. 46 Maurenbrecher $=51$ La Penna - Funari), citato da Festo (p. 210 Lindsay): magnis operibus perfectis obsidium coepit per L. Catilinam legatum ${ }^{2}$.

(1) Queste notizie rappresentano un primo nucleo della "leggenda nera" di Catilina, creato ad arte da Cicerone durante la campagna elettorale del 64, in cui Catilina fu suo avversario. Sulla natura almeno in parte calunniosa di questa tradizione e sui fraintendimenti che ne caratterizzarono l'elaborazione più tarda, mi riservo di tornare in altra sede.

(2) Questo è il testo trasmesso dal codice Farnesianus IV.A.3 (xI secolo), riprodotto sia nell'edizione Lindsay di Festo (Leipzig, 1913), sia nella recente edizione La Penna - Funari del primo libro delle Historiae (Berlin - Boston, 2015). 
Nel secolo scorso, il contesto dell'episodio cui il frammento allude è stato per lungo tempo oggetto di discussione; ma in questi ultimi decenni una sostanziale unanimità pare raggiunta: l'assedio (obsidium) citato da Sallustio viene in genere riferito alla guerra civile sillana. In questo breve studio mi propongo di rimettere in discussione tale ipotesi. Credo infatti che essa non poggi su basi solidissime e che diverse considerazioni possano suggerire una ricostruzione alternativa.

Il frammento parla di un ignoto personaggio il quale, dopo aver terminato imponenti lavori preparatori, ordina di dare inizio a un assedio e ne affida il compito al legatus Catilina. Sul luogo e sulla data di tale assedio sono state avanzate diverse ipotesi: in Italia, nella prima metà degli anni 80; in Italia, sotto Silla, nell'82 o poco dopo; fuori d'Italia, negli anni 70.

La cronologia alta fu proposta da Pareti, nella sua biografia catilinaria del 1934: secondo Pareti, l'obsidium di cui parla Sallustio sarebbe un episodio della guerra sociale (90-88) o della guerra civile dell' $87-86^{3}$; se ammettiamo la prima ipotesi, il personaggio non menzionato, soggetto di coepit, sarebbe identificabile con Cn. Pompeo Strabone, console dell'89. In effetti, un legame tra i due personaggi è suggerito dalla nota iscrizione $\left(C I L, \mathrm{I}^{2}, 709=\mathrm{VI}, 37045=I L S 8888=I L L R P\right.$ 515) contenente l'elenco dei membri del consilium di Pompeo Strabone durante l'assedio di Ascoli: tra essi, alla linea 11, è menzionato un L. Sergius L. f. Tro., che va molto probabilmente identificato con Catilina ${ }^{4}$. Ma l'ipotesi di Pareti non ha avuto molto seguito ed è oggi giustamente esclusa: nella prima metà degli anni 80 , Catilina sarebbe stato decisamente troppo giovane ${ }^{5}$ per assumere la funzione di legatus ${ }^{6}$.

Più interessante, e fortunata, è senz'altro l'ipotesi che vede in questo assedio un episodio della guerra civile sillana. Essa fu sostenuta nel 1893 da Maurenbrecher, nella sua edizione delle Historiae: il frammento di Sallustio alluderebbe all'assedio di Preneste dell'82; Catilina vi avrebbe preso parte in qualità di legatus e avrebbe portato a Preneste la testa tagliata dei leader marianisti, perché fossero mostrate agli assediati ${ }^{7}$. Quest'ultimo dettaglio è stato ammesso, tra gli altri, anche da Gelzer e da Broughton ${ }^{8}$; ma l'ipotesi è priva di riscontro, poiché le due fonti che accennano alla macabra operazione (App., BC, 1.93.433; Oros., hist., 5.21.8) non menzionano Catilina.

Trent'anni dopo la sua formulazione, l'ipotesi di Maurenbrecher fu oggetto di un'articolata critica da parte di Cichorius ${ }^{9}$, che possiamo sintetizzare nei seguenti punti:

1. non è verosimile l'avanzamento di Catilina da miles a legatus in soli sette anni, ossia dall' 89 (anno cui risale l'iscrizione di Ascoli) all'82 (anno dell'assedio di Preneste);

2. secondo l'esplicita testimonianza di Appiano $(B C, 1.88 .402)$, l'assedio di Preneste fu condotto sin dall'inizio da Q. Lucrezio Afella;

3. le parole utilizzate nel frammento (obsidium coepit) suggeriscono che nel testo originale perduto questo assedio fosse descritto dettagliatamente. Non si può perciò attribuirlo con certezza alla sezione introduttiva del primo libro delle Historiae (dedicata appunto alla

(3) PARETi 1965, p. 294-295.

(4) L'identificazione è generalmente ammessa: cfr., tra gli altri, Cichorius 1922, p. 172; Gelzer 1923, c. 1693; PARETI 1965, p. 293; Manni 1969, p. 21; Suolahti 1955, p. 339; Syme 1964, p. 65; Criniti 1970, p. 160-162; più recentemente FezZi 2013, p. 9; Santangelo 2014, p. 4; Levick 2015, p. 1-2; Schietinger 2017, p. 161.

(5) La data di nascita di Catilina è ignota. Tenuto conto dei limiti d'età fissati dalla lex Cornelia de magistratibus, poiché egli fu pretore nel 68, non può essere nato dopo il 108 (Drumann - Groebe 1919, p. 414; Cichorius 1922, p. 172-173; Gelzer 1923, c. 1693; PARETi 1965, p. 291; MANNi 1969, p. 21).

(6) Questa obiezione resta valida anche se l'opinione, un tempo diffusa (Premerstein 1924, c. 1141; Veith, in Kromayer - Veith 1928, p. 398; Sullahti 1955, p. 13, 27; Harmand 1967, p. 369), secondo cui i legati erano sempre senatori è in realtà priva di fondamento (Smith 1958, p. 62-64; Schleussner 1978, p. 152-153; Keaveney - Strachan 1981, p. 364).

(7) Maurenbrecher 1893, p. 16.

(8) Gelzer 1923, c. 1694; Broughton 1952, p. 72. Cfr. anche Thomasson 1991, p. 110.

(9) Cichorius 1922, p. 173. 
guerra civile). È anzi più probabile che esso facesse parte della narrazione vera e propria, che iniziava, come è noto, dal 78 ( $1 \mathrm{fr} .1:$ M. Lepido Q. Catulo consulibus);

4. se è così, la notizia di Sallustio dovrebbe riguardare uno dei conflitti extra-italici successivi alla morte di Silla.

La cronologia "bassa" proposta da Cichorius ha goduto di un seguito abbastanza limitato (ci ritorneremo tra poco) e si è infine imposta l'opinione che fa di Catilina un legatus di Silla, nell'82 o poco dopo ${ }^{10}$. A questo proposito, merita un'attenzione particolare un articolo di Keaveney e Strachan $\left[=\right.$ K./S.], pubblicato nel $1981^{11}$. In questo articolo, gli argomenti di Cichorius sono oggetto di una critica puntuale, articolata come segue:

1. non è vero che sette anni costituissero un periodo di tempo troppo breve per diventare legatus, né che fosse obbligatorio, per ottenere questa carica, essere senatori ${ }^{12}$;

2. perché Catilina divenisse legatus, era sufficiente la volontà di Silla;

3. è in ogni caso molto probabile che Catilina fosse uno dei 300 senatori "sillani";

4. non si può considerare un frammento di nove parole come un racconto dettagliato ${ }^{13}$;

5. K./S. aggiungono inoltre, contro Maurenbrecher, che il frammento potrebbe alludere a qualsiasi assedio della guerra civile, dato che le operazioni in Italia continuarono in diverse zone ancora dopo l'82 (Liv., perioch. 89; Gran. Lic. 36.8-9).

Si può senz'altro concordare con K./S. sui punti (1) e (2). Quanto al punto (3), che Catilina sia entrato in senato già in occasione della lectio dell' 81 resta, tutto sommato, improbabile ${ }^{14}$; ma non si tratta comunque di un problema rilevante per la contestualizzazione del frammento. Sul punto (4), K./S. hanno ragione in linea di principio, ma Cichorius diceva che dalle parole del frammento si ricava che il suo contesto narrativo fosse dettagliato. Sul punto (5), infine, l'obiezione di K./S. è pienamente fondata: non c'è in effetti alcun motivo che obblighi a identificare l'obsidium del frammento con l'assedio di Preneste.

Noi sappiamo molto poco sulle operazioni condotte in Italia dopo l'82 e una localizzazione certa dell'episodio non è in ogni caso possibile ${ }^{15}$. La proposta di K./S. è di identificare la città assediata con Isernia, che era stata occupata dagli Italici nel 90 (Liv., perioch. 72-73; App., BC, 1.41.182; Diod. 37 fr. 2.9 Dindorf [= Phot., bibl., 391b]; fr. 19.1-2 Dindorf = 26-27 Goukowsky). Ma diverse considerazioni inducono a respingere questa identificazione. Anzitutto, la cronologia e le modalità della riconquista di Isernia restano sconosciute, poiché nessuna fonte vi accenna. Inoltre (ed è questo il punto cruciale), la proposta di K./S. si basa su una correzione della periocha 89 di Livio: Sylla Nolam in Samnio recepit. In questo passo, la localizzazione nel Sannio della città campana di Nola è un evidente errore, ma la lezione in Samnio è attestata da quasi tutta la tradizione. La sola

(10) Cfr. per esempio Broughton 1952, p. 72; Criniti 1970, p. 161; Criniti 1990, p. 20; Angeli Bertinelli 1997, p. 395 (e, implicitamente, La Penna 1963, p. 11).

(11) Keaveney - Strachan 1981.

(12) Cfr. supra, n. 6.

(13) Keaveney - Strachan 1981, p. 364.

(14) L'innalzamento a 30 anni dell'età minima per accedere alla questura, stabilito nell' 81 dalla lex Cornelia de magistratibus, implicava quello dell'età minima per entrare in senato. Il caso di Pompeo, che nell'81 aveva 25 anni e che fu iscritto in senato solo in occasione della lectio del 70 (Val. Max. 8.15.8; Plin., nat., 7.26.96; Plut., Pomp., 23.2; Cass. Dio $36.25 .2 ; 36.27 .4)$, ci fornisce un termine di confronto. Per essere iscritto tra i senatori nell' 81 , Catilina avrebbe dovuto essere nato, al più tardi, nel 111: questo non è di per sé impossibile, ma appare improbabile, se teniamo conto degli indizi sparsi nella sua biografia. Sulla composizione del senato di Silla, cfr. Willems 1878, p. 401-415; Hill 1932; GabBa 1951, p. $262-270=1973$, p. 159-172; GABBA $1956=1973$, p. 407-425; HAWTHORN 1962; NiCOLET 1966, p. 573-580; HANTOS 1980, p. 45-61; Develin 1987; Santangelo 2006; Steel 2014a; Steel 2014b. Nessuno degli studi citati menziona Catilina tra i possibili senatori "sillani".

(15) Lo rilevava già MAURenbrecher 1893, p. 18: Coniecturis opus esse nemo non concedet. 
eccezione è quella del codice Nazarianus (Palatinus Latinus Heidelbergensis 894, del Ix secolo) ${ }^{16}$ : esso riporta il testo Syllam tam in Samnio recepit, che non significa niente. Mommsen ${ }^{17}$ correggeva radicalmente (e arbitrariamente): Sylla Aeserniam in Samnio recepit. K./S. vanno ancora più in là e propongono di leggere: Sylla Nolam <et Aeserniam > in Samnio recepit. Certo, i due studiosi non si limitano a proporre questa integrazione: essi cercano di giustificarla, mostrando che questo codice contiene diversi errori analoghi. Ma l'impressione di una "violenza" inflitta al testo rimane. L'ipotesi che si sia passati da Nolam et Aeserniam (testo originale della periocha secondo K./S.) a tam non solo inserisce un ulteriore elemento (et Aeserniam) rispetto all'ipotesi comunemente ammessa (Nolam > tam), ma sembra avere l'unico (e discutibile) obiettivo di correggere l'incongruenza "geografica" del testo, che potrebbe benissimo essere un errore di Livio o, meglio, dell'anonimo redattore delle periochae.

È vero che nel loro articolo K./S. sottolineavano opportunamente che la loro correzione restava un'ipotesi («must remain speculative») ${ }^{18}$. Tuttavia, questa prudenza fu sacrificata alla sintesi l'anno dopo, nella prima edizione dell'eccellente biografia sillana di Keaveney («Aesernia, also, was taken in 79 by a force under the command of Catiline» $)^{19}$. Nel 1986, Broughton accettò questa ricostruzione nel volume di addenda ai Magistrates of the Roman republic ${ }^{20}$ : in questo modo, quella che cinque anni prima era presentata come un'ipotesi divenne, per così dire, la versione "canonica". Come tale, essa fu accolta nel 1992 da McGushin, nel suo commento ai frammenti delle Historiae ${ }^{21}$. L'incongruenza è stata infine eliminata dallo stesso Keaveney nel 2005, nella seconda edizione del Sulla: «In 80 Nola and Aesernia, which may have been resisting since the Social War, surrendered. It is possible Sulla himself directed operations at Nola while his legatus Catilina had charge at Aesernia» ${ }^{22}$.

Una simile ipotesi (come già quella di Maurenbrecher) si basa sul presupposto che Catilina non fosse solo legatus, ma che lo fosse agli ordini di Silla: "Given the conspicuous loyalty, not to say ferocity which Catiline had shown in the Sullan cause it would be by no means surprising if Sulla had made some further use of him. The killing of Gratidianus was perhaps the most atrocious, and certainly the most notorious of the services Catilina had rendered, but our sources leave us in no doubt that there had been many others of a like kind. He could plainly be counted upon to deal with appropriate ruthlessness with the opponents of Sulla wherever they might be» ${ }^{23}$. Non è questa la sede per approfondire il tema della "ferocia" di Catilina (ivi compresa l'esecuzione collettiva di Gratidiano, che non fu un episodio isolato e di cui Catilina non fu il solo responsabile $)^{24}$. Quel che conta rilevare è che si tratta in fondo di una versione più "ciceroniana" di Cicerone: Catilina dovette senza dubbio rendersi colpevole di atti di crudeltà ancora più numerosi di quelli di cui parlano le fonti. E a questo contesto andrebbe riferito anche l'assedio da lui condotto in qualità di legatus: "Whether or not this reconstruction is accepted the fact remains that Catiline is definitely stated legatus in the Sallust fragment and thus can surely only be the legate of the consul Sulla in $80 »^{25}$.

(16) Su questo codice cfr. JAL 1984, xcix: «En dépit de son ancienneté et de sa qualité [...] N n’en contient pas moins un certain nombre de leçons aberrantes».

(17) Citato da Keaveney - Strachan 1981, p. 366. Cfr. anche Hülsen 1894a, c. 1300.

(18) Keaveney - Strachan 1981, p. 365-366.

(19) KeAveney 1982, p. 199.

(20) Broughton 1986, p. 192.

(21) McGushin 1992, p. 110-112.

(22) Keaveney 2005a, p. 163. Si ritrova la stessa prudenza in Keaveney 2005b, p. 437-438; La Penna, in La Penna FUNARI 2015, p. 166.

(23) Keaveney - Strachan 1981, p. 364.

(24) Hinard 1984, p. 302-307 = 2011, p. 92-95; Hinard 1986 = 2011, p. 143-146; Marshall 1985; Urso c.d.s.

(25) Keaveney 2005b, p. 438 
Che Catilina, se fu legatus, lo sia stato necessariamente sotto le insegne di Silla è in realtà un falso presupposto. Si tratta in definitiva dello stesso equivoco che già nel 1893 ispirava Maurenbrecher e che si risolveva in una petizione di principio. Il commento dello studioso tedesco al frammento sallustiano appare a questo proposito rivelatore: Fragmento difficili rem in oppugnatione oppidi alicuius gestam referri apparet, de bello civili agi veri simillimum est, quippe quo Catilina Sullae legatus fuerit ${ }^{26}$. Insomma, Maurenbrecher attribuiva questo assedio agli anni della guerra civile perché Catilina era stato legatus di Silla; ma la sola notizia su Catilina legatus è appunto questo frammento di Sallustio, in cui il nome di Silla non compare!

Come è noto, il primo libro delle Historiae, dopo un'introduzione sulla guerra sociale e la guerra civile (fr. 15-53 M. = 21-51 L.P./F.), comprendeva il racconto degli avvenimenti del 78 e di una parte del 77: era insomma sostanzialmente dedicato al tumultus Lepidi e all'inizio del bellum Sertorianum ${ }^{27}$. L'introduzione sugli anni 80 doveva essere relativamente breve ${ }^{28}$, tanto più che Sallustio aveva affermato, nel Bellum Iugurthinum (95.4), di non avere intenzione di occuparsi ulteriormente di Silla ${ }^{29}$.

In linea di principio, dovrebbe perciò essere più probabile che il frammento facesse originariamente parte della narrazione sul biennio 78-77, piuttosto che di quella sugli anni 80 . E in effetti non sono mancati, in passato, sostenitori di questa cronologia "bassa", che hanno proposto diverse possibili contestualizzazioni del nostro obsidium ${ }^{30}$ : la spedizione di Ap. Claudio Pulcro contro i Traci (cfr. Sall., hist., 1 fr. 133-134 M. = 127-128 L.P./F.) ${ }^{31}$; la campagna di P. Servilio Vatia Isaurico contro i pirati in Cicilia (fr. 127-132 M. = 122-126 L.P./F.) ${ }^{32}$; quella di Q. Cecilio Metello Pio contro Sertorio in Spagna (fr. 84-126 M. = 77-121 L.P./F.) ${ }^{33}$; o addirittura una spedizione di C. Scribonio Curione, proconsole in Macedonia nel 75 (ma non si capisce come Sallustio avrebbe potuto parlarne nel primo libro delle Historiae $)^{34}$. Nessuno è stato in grado di proporre argomenti concreti a sostegno delle rispettive ipotesi ${ }^{35}$.

(26) Maurenbrecher 1893, p. 18.

(27) La Penna 1963, p. 12-15; La Penna, in La Penna - Funari 2015, p. 47. E cfr. anche Cichorius 1922, p. 173.

(28) Nell'edizione La Penna - Funari, su 146 frammenti del primo libro, 6 sono attribuiti alla guerra sociale (fr. 21-26), 25 (fr. 27-51) alla guerra civile.

(29) Rawson 1987, che parla in un primo tempo di una «fairly brief introduction to his main narrative» (p. 167), afferma in seguito che questa introduzione doveva essere abbastanza estesa: «A comparatively extensive preliminary account of the eighties» (p. 178).

(30) Cfr., tra gli altri, Syme 1964, p. 65-66: «At some time early in the seventies he had a service as a legatus abroad, under a proconsul in one of the many wars».

(31) Drumann - Groebe 1919, p. 417

(32) Cichorius 1922, p. 173-174.

(33) Havas 1974.

(34) Ipotesi citata da CRINITI 1990, p. 295.

(35) In particolare, l'argomentazione di Havas (cfr. supra, n. 33) si basa sul fatto che «dans le matériel épigraphique d'Hispanie, le nom de Sergius apparait fréquemment». Egli cita in particolare CIL, II suppl., in cui il nomen Sergius «est relevé dans 21 cas, dont 19 fois avec certitude». Ma questo dimostra ben poco, tanto più che (come Havas stesso riconosce) i Sergii sembrano avere intrattenuto rapporti col mondo iberico fin dalla seconda guerra punica. A partire da queste premesse di per sé fragili, Havas formula in seguito ulteriori ipotesi, come quella secondo cui Catilina avrebbe avuto rapporti difficili con il suo presunto comandante in Spagna, Metello Pio: queste difficoltà sarebbero suggerite, se non dimostrate, dall'appoggio prestato da Metello agli Africani nel 66, quando essi accusarono de repetundis il loro ex governatore Catilina (Cic. in Asc., tog. cand., p. 87 Clark). Ma i legami tra i Metelli e la provincia d'Africa sono ben noti e non è certo necessario immaginare un'antipatia personale di lunga data per spiegare l'iniziativa di Metello Pio. Havas ritiene inoltre significativo il fatto che C. Cornelio Cetego, uno dei congiurati del 63, abbia ferito Metello in Spagna: ma questa accusa è menzionata soltanto da Cicerone, nella Pro Sulla (25.70) pronunciata nel 62, quando sia Metello sia Cetego erano morti. Infine, secondo Havas, «il n'est pas exclu» che l'accusa contro Catilina nel 73, nell'affare delle Vestali (Q. Cic., pet., 10; Cic. in Asc., tog. cand., p. 91 Clark; Asc., ibid.; Sall., Catil., 15.1; Oros., hist., 6.3.1), sia il frutto «d'une action concertée des Caecilii Metelli»: ma nessun argomento è fornito a sostegno di questa ipotesi. 
A questa lista di ipotesi "indimostrabili", vorrei aggiungerne a mia volta una. Se l'assedio di cui parlava Sallustio deve essere più probabilmente attribuito agli anni 78-77, non è necessario cercarne la localizzazione fuori dall'Italia. Proprio in occasione del tumultus Lepidi, infatti, diverse operazioni furono condotte contro i sostenitori del console del 78, sotto il comando del suo ex collega Q. Lutazio Catulo e di Pompeo. Tra queste operazioni la tradizione annovera almeno due assedi.

Il primo è l'assedio di Modena, condotto da Pompeo contro M. Giunio Bruto (tribuno della plebe nell'83 e padre del cesaricida), in seguito ucciso a tradimento. Livio parlava certamente di questa morte (cfr. perioch. 90); Cicerone (Att., 9.14.2), Valerio Massimo (6.2.8), Appiano (BC, 2.111.464) e Orosio (hist., 5.22.17) le dedicano brevi cenni; ma la fonte principale è Plutarco, nella Vita di Bruto (4.1-3) e soprattutto nella Vita di Pompeo (16.3-7), dove si trova il solo passo che ci descriva l'assedio. Secondo Plutarco, Pompeo fu nominato «comandante della spedizione

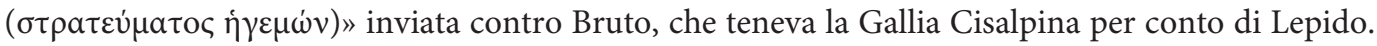
Pompeo «si impadronì facilmente della maggior parte delle città della regione, tranne Modena, in

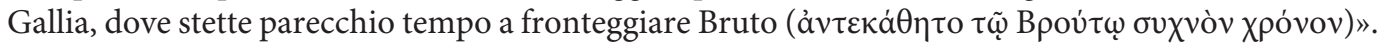
Dunque, secondo Plutarco l'assedio di Modena durò "parecchio tempo» e fu preceduto dalla rioccupazione delle altre città della zona ${ }^{36}$. Come è raccontato da Plutarco, l'attacco a Modena potrebbe ben essere definito con il termine obsidium che troviamo nel frammento di Sallustio. Del resto, Sallustio parlava certamente dell'attacco di Pompeo a Modena: l'espressione apud Mutinam, che Prisciano leggeva nel primo libro delle Historiae (gramm., 67.2 = Sall., hist., 1 fr. 79 M. = 73 L.P./F.), non può che riferirsi a questo episodio ${ }^{37}$.

Ma ci fu anche un secondo assedio, di cui parla Orosio (hist., 5.22.17):

Albanorum civitas, obsidione oppugnata atque excruciata fame ultima, miserabilium reliquiarum deditione servata est; ubi tunc Scipio, Lepidi filius, captus atque occisus est. Brutus in cisalpinam Galliam fugiens persequente Pompeio apud Regium interfectum est.

Già Hülsen aveva identificato questa civitas Albanorum con Alba Fucens ${ }^{38}$. La sua ipotesi è stata però respinta per lungo tempo: pareva difficilmente ammissibile che tanti partigiani di Lepido si fossero riuniti in un luogo così vicino a Roma. Si è dunque proposto di identificare questa città con Alba Pompeia (l'odierna Alba, in Piemonte) ${ }^{39}$. Ma contro questa ipotesi si è infine pronunciato Coarelli nel $1998^{40}$, nell'ambito di una nuova interpretazione della "Terrazza Nord" di Alba Fucens come la tomba di L. Cornelio Scipione Emiliano, il Lepidi filius citato da Orosio ${ }^{41}$ : Coarelli ha dimostrato con argomenti inoppugnabili che l'Albanorum civitas era sicuramente Alba Fucens ${ }^{42}$.

(36) Tra cui certamente Milano, come suggerisce Frontin., strat., 1.9.3.

(37) Per quanto molto breve, il contesto del frammento sembra certo: non si vede per quale altra ragione Sallustio avrebbe dovuto menzionare la città di Modena nel primo libro delle Historiae.

(38) HüLSEN 1894b.

(39) Nel contesto di questa ipotesi, anche il seguito delle operazioni, descritto da Plutarco e da Orosio, è stato oggetto di discussione: se si ammette che Pompeo abbia condotto personalmente i due assedi, egli avrebbe probabilmente dovuto marciare dapprima contro Modena, per poi dirigersi verso Alba Pompeia (Criniti 1969a, p. 438; Criniti 1969b, p. 872; Labruna 1975, p. 175; Heftner 1994, p. 135). Tuttavia Orosio non attribuisce esplicitamente a Pompeo la conduzione di questo assedio: ciò che ha indotto già Holmes 1923, p. 369 e in seguito Heftner 1994, p. 135 ad avanzare l'ipotesi secondo cui le operazioni ad Alba Pompeia sarebbero state affidate a un legatus.

(40) Coarelli 1998.

(41) Questa tomba fu costruita da Lepido, il futuro triumviro, nel 49, nel momento in cui egli prese ai pompeiani la città di Alba Fucens.

(42) Tra gli argomenti utilizzati da Coarelli, mi limito a citare qui un passo di Orosio, fino allora sfuggito all'attenzione degli studiosi. Due capitoli dopo la menzione dell'Albanorum civitas, Orosio scrive (5.24.16): nam Lepidus et Scipio in Italia, Brutus in Gallia, Domitius Cinnae gener in Africa, Carbo in Cossura et Sicilia, Perpenna in Liguria et post cum Sertorio in Hispania [...] haec tunc civilia, vel quo alio dicenda sunt nomine, bella excitantes, de uno multa, de magno magna fecerunt. Qui Orosio fa una distinzione molto chiara tra le operazioni condotte contro Lepido e suo figlio in Italia e quelle contro 
Riassumendo: il frammento di Sallustio su Catilina legatus, tratto dal primo libro delle Historiae, riguarda l'assedio di una città sconosciuta; questo assedio è stato identificato con operazioni di guerra condotte presso Isernia dopo l'82 - operazioni che nessuna fonte descrive; il primo libro delle Historiae era incentrato sul biennio 78-77 e sul tumultus Lepidi; durante il tumultus Lepidi, sono esplicitamente attestati due assedi: quello di Modena (contro Bruto) e quello di Alba Fucens (contro il figlio di Lepido). Tenuto conto di questi elementi, non mi pare troppo azzardato di proporre l'identificazione con Modena o con Alba Fucens della città assediata da Catilina legatus. Delle due possibilità, la più probabile parrebbe appunto Alba Fucens. Non si vede infatti la ragione per cui Pompeo avrebbe dovuto attendere prima di attaccare Bruto a Modena e delegare a tale scopo un legatus. Al contrario, sul fronte meridionale (Etruria e Lazio) le forze "lealiste" dovevano fronteggiare Lepido e suo figlio Scipione. Catulo, che era il comandante (almeno) di questo fronte, fece certamente ricorso ad alcuni legati per contrastare Scipione, mentre lui era impegnato contro Lepido. Tra questi potrebbe esserci stato anche Catilina.

Se ammettiamo l'ipotesi "Modena", Catilina sarebbe stato legatus di Pompeo (se si ritiene che quest'ultimo disponesse di un imperium pro praetore) o di Catulo (se si pensa che Pompeo fosse, lui pure, legatus) $)^{43}$; se ammettiamo l'ipotesi “Alba Fucens", egli sarebbe stato senz'altro legatus di Catulo. L'uno e l'altro lo conoscevano bene. Nell'89 Pompeo e Catilina erano stati commilitoni al campo di Ascoli e membri del consilium di Pompeo Strabone, padre di Pompeo ${ }^{44}$; quest'ultimo aveva potuto constatare sul campo le qualità militari di L. Sergius (Cic., Cael., 5.12; 6.13; Phil., 2.5.9; Sall., Catil., 5.1-3; 61.4) e lo aveva in seguito ritrovato tra i partigiani di Silla. Il legame di Catilina con Catulo, esponente dell'ala più "conservatrice" degli optimates, è molto più complesso. Tale legame era nato al più tardi nel novembre dell'82, quando Catilina aveva catturato Gratidiano, che cinque anni prima era stato responsabile del suicidio del padre di Catulo (console del 102 ed eroe, col suo rivale Mario, della guerra cimbrica), e lo aveva condotto alla tomba di quest'ultimo (Q. Cic., pet., 10; Val. Max. 9.2.1; Sen., dial., 5.18.2; cfr. Luc. 2.173-174) perché vi fosse massacrato. I rapporti tra Catilina e Catulo sono ben attestati anche in seguito: nel $73 \mathrm{fu}$ proprio l'intervento di Catulo, in qualità di membro anziano del collegio dei pontefici ${ }^{45}$, a evitare a Catilina una possibile condanna de incestu a seguito dello scandalo delle Vestali (Oros., hist., 6.3.1); e nel novembre del 63, dopo avere lasciato Roma per andare incontro al suo destino, Catilina scrisse appunto a Catulo la sua ultima lettera, affidando alla protezione del vecchio amico l'incolumità di sua moglie Orestilla (Sall.,

Bruto in Gallia, cioè a Modena: l'affermazione della nostra fonte è chiara ed esplicita, e induce a concludere che l'Albanorum civitas, dove Scipione fu assediato e poi ucciso, era appunto Alba Fucens. Inoltre Coarelli mette giustamente in evidenza che la sequenza degli avvenimenti proposta da Orosio (nella quale l'Albanorum civitas è presa prima della morte di Bruto) è stata invertita senza motivo nelle ricostruzioni dei moderni e che l'occupazione di Alba Pompeia (fondata da Pompeo Strabone) da parte dei nemici di Pompeo (suo figlio) è in sé più "inverosimile" della presenza di forze fedeli a Lepido ad Alba Fucens. Le conclusioni di Coarelli sono state accolte da Allély 2004, p. 65-68; Allély 2008, p. 614; Santangelo 2014, p. 18-20. Етснето 2012, p. 185 cita le diverse identificazioni del sito, senza però pronunciarsi.

(43) Sui poteri di Pompeo durante la guerra contro Lepido, all'opinione di chi lo considera come un legatus pro praetore subordinato a Catulo (cfr. Hillman 1998; Girardet 2001, p. 165-166), si oppone quella di chi ritiene che egli detenesse un imperium pro praetore del tutto indipendente (cfr. la convincente dimostrazione di VERVAET 2009; rinvio agli studi di Hillman e di Vervaet per la bibliografia precedente). Come Vervaet ha mostrato (p. 407-408), l'affermazione di Plutarco (Pomp., 16.2) secondo cui Catulo era collega ( $\sigma v v \alpha ́ \rho \chi \omega v)$ di Lepido suggerisce che Pompeo esercitasse il suo comando già nel 78.

(44) L'iscrizione di Ascoli menziona, alla linea 8, Cn. Pompeius Cn. f. Clu. Sull'identità del personaggio non ci sono dubbi.

(45) In assenza del pontefice massimo Metello Pio, impegnato in Spagna contro Sertorio. Catulo è il primo dei pontefici menzionati nella lista di Macrobio (sat., 3.13.11), che si riferisce agli anni tra il 74/3 (cooptazione di Cesare) e il 69 (cooptazione di P. Sulpicio Galba) e che sembra ordinata per anzianità (TAYLOR 1942, p. 400; Broughton 1952, p. 114; LEWIS 2001, p. 144; CADOUX 2005, p. 167). 
Catil., 35) ${ }^{46}$. Non è forse un caso se il giovane L. Sergius (Silus) assunse (o ricevette) il soprannome di Catilina, che mai nessun membro della gens Sergia aveva portato prima di lui ${ }^{47}$ : questo soprannome deriva da Catulina caro ${ }^{48}$, ossia "carne di cagnolino" - in latino appunto catulus.

Nel momento del tumultus Lepidi, Catulo e Pompeo apparivano come i difensori dell'eredità sillana (Flor. 2.11.6: Sullanae dominationis duces atque signiferi) ${ }^{49}$. L'eventuale partecipazione di Catilina al conflitto in qualità di legatus di Catulo (o di Pompeo) manterrebbe (paradossalmente) l'implicazione "sillana" del frammento, ma senza fare di Catilina un legatus di Silla. Essa confermerebbe che l'adesione di Catilina alla fazione di Silla non fu una scelta opportunista e provvisoria, come hanno creduto in molti ${ }^{50}$, ma che fu accompagnata da un comportamento coerente, prima e dopo la morte del dittatore. Catilina aveva combattuto durante la guerra sociale agli ordini del padre di Pompeo; aveva in seguito vendicato il padre di Catulo; dopo Silla, questi legami non furono interrotti: ancora nel 64, questo passato sillano, che Catilina si guardava bene dal rinnegare (cfr. Cic. in Asc., tog. cand., p. 90 Clark) ${ }^{51}$, fu al centro degli attacchi rivoltigli da Cicerone, suo rivale alle elezioni consolari, e Catilina fu imputato in uno dei processi presso la quaestio de sicariis istruiti su iniziativa di Cesare (Cass. Dio 37.10.2-3; cfr. Cic., Lig., 4.12; Suet., Iul., 11.2; scholia Gronoviana, p. 293 Stangl $)^{52}$.

Vale la pena di concludere con un accenno alle convulse e drammatiche giornate di fine ottobre/ inizio novembre del 63. Il 27 ottobre scoppiò in Etruria l'insurrezione degli ex-veterani di Silla. Si trattava degli stessi veterani che un mese prima, o poco più, si erano recati a Roma per appoggiare l'ultima candidatura di Catilina al consolato ${ }^{53}$ : il console Cicerone aveva allora interpretato a modo suo il loro arrivo nell'urbe, accusando Catilina di avere propositi sovversivi e chiedendo (invano) che il senato si pronunciasse contro di lui (Cic., Mur., 25.51). Nel momento in cui i veterani si ribellarono, molti videro in Catilina l'ispiratore di questa rivolta: si cominciò a istruire un processo contro di lui, in virtù della lex Plautia de vi (Sall., Catil., 31.4; Cass. Dio 37.31.3) ${ }^{54}$. Fu un certo L. Emilio Paolo a denunciarlo. L'identità di questo personaggio non sembra priva di interesse: egli va infatti identificato con il fratello del triumviro Lepido (Vell. 2.67.3; Plut., Ant., 19.3; Flor. 2.16.4; App., BC, 4.12.45; Cass. Dio 47.6.3; Oros., hist., 6.18.11), il che fa di lui il figlio del console "sovversivo" del $78 !^{55}$ Come spesso accadeva in questi casi, l'accusa era rappresentata da un giovane a inizio carriera, desideroso di farsi notare; ma se davvero l'obsidium guidato da L. Catilina

(46) L'analisi lessicale e stilistica di questo capitolo ha mostrato che esso non è una libera composizione di Sallustio: si tratta, se non del testo originale della lettera, di una sua riproduzione abbastanza fedele (HoffmanN 1959, p. 473; Syme 1964, p. 71-72; Earl 1966, p. 94-95; Mazzarino 1966, p. 381; McGushin 1977, p. 195-196; Ramsey 2007, p. 155; Bringmann 2010, p. 93; FezZI 2013, p. 42).

(47) La sua prima attestazione sicura è contenuta nella prima lettera di Cicerone ad Attico del luglio 65 (Att., 1.1.1: Catilina, si iudicatum erit meridie non lucere, certus erit competitor).

(48) Wölfflin 1884; Отto 1907-1913, c. 259; Pareti 1965, p. 292; Niedermann 1935, p. 276-278; Walde Hoffmann 1982, p. 183; Kajanto 1965, p. 91, 340; Alföldi 1966, p. 713; Ramsey 2007, p. 68; Hinard 1985, p. 19. Cfr. anche Ernout - Meillet 1959, p. 106; Criniti 1990, p. 19.

(49) Floro utilizza questa espressione appunto nel momento in cui tratta gli avvenimenti del 77. Su Catulo in particolare, cfr. BuRton 2014, p. 409: «Catulus [...] represented himself as the standard-bearer of Sullan orthodoxy».

(50) Cfr., tra gli altri, Badian 1962, p. 60; Syme 1964, p. 86; Bessone 2004, p. 141; Keaveney 2005a, p. 129.

(51) Tu tibi ne infitiandi quidem impudentiae locum reliquisti. Secondo Appiano, del resto, Catilina non era stato solo

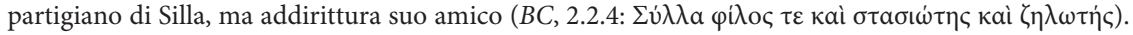

(52) Basterebbe questo fatto per smentire la favola di un'intesa politica tra Catilina e Cesare: una calunnia postuma di Cicerone (che ne scrisse nel De consiliis suis: Asc., tog. cand., p. 83 Clark; Plut., Crass., 13.4; Cass. Dio 39.10.2-3), alla quale hanno prestato fede molti studiosi moderni.

(53) Che le elezioni del 63 si siano svolte in settembre è stato ora dimostrato in modo persuasivo da RAMSEY 2019.

(54) Che il minacciato processo riguardasse i fatti d'Etruria e non la "congiura" risulta chiaramente da Dione.

(55) KLEBS 1894, c. 564. 
legatus era stato un episodio del tumultus Lepidi, l'iniziativa di L. Emilio Paolo contro Catilina si spiegherebbe come un classico esempio di vendetta familiare.

Gianpaolo Urso

Università Cattolica del Sacro Cuore - Milano

\section{Bibliografia}

Alföldi, A., 1966, «Les cognomina des magistrats de la République romaine», in R. Chevallier (ed.), Mélanges d'archéologie et d'histoire offerts à André Piganiol, II, Paris, p. 709-722.

Allély, A., 2004, Lépide le triumvir, Bordeaux.

AlléLy, A., 2008, «Le sort des enfants des hostes publici à Rome à la fin de la République. L'exemple des Aemilii Lepidi», Athenaeum 90, p. 609-622.

Angeli Bertinelli, M. G., Manfredini, M., Piccirilli, L., Pisani, G., 1997, Plutarco. Le vite di Lisandro e di Silla, Milano.

BADIAN, E., 1962, «Waiting for Sulla», JRS 52, p. 47-61.

Bessone, L., 2004, Le congiure di Catilina, Padova.

Bringmann, K., 2010, Cicero, Darmstadt.

Broughton, T.R.S., 1952, The magistrates of the Roman republic, II, New York.

Broughton, T.R. S., 1986, The magistrates of the Roman republic, III, Atlanta.

Burton, P., 2014, «The revolt of Lepidus (cos. 78 BC) revisited», Historia 63, p. 404-421.

CAdoux, T.J., 2005, «Catiline and the Vestal virgins», Historia 54, p. 162-179.

Cichorius, C., 1922, Römische Studien, Leipzig - Berlin.

CoArelli, F., 1998, «Lépide et Alba Fucens», REA 100, p. 461-475.

Criniti, N., 1969a, «M. Aimilius Q.f. M.n. Lepidus 'ut ignis in stipula'», MIL 30, p. 319-460.

Criniti, N., 1969b, «Tre noterelle di storia lepidana», RIL 103, p. 865-874.

Criniti, N., 1970, L'epigrafe di Asculum di Gn. Pompeo Strabone, Milano.

CRINITI, N., 1990, «Catilina: cognomen atque omen?», in Tradizione dell'antico nelle letterature e nelle arti d'occidente. Studi in memoria di Maria Bellincioni Scarpat, Parma, p. 16-29.

Develin, R., 1987, «Sulla and the Senate», AHB 1, p. 130-133.

Drumann, W., Groebe, P., 1908-1919, Geschichte Roms, IV-V, Leipzig.

EARL, D. C., 1966, The political thought of Sallust, Amsterdam.

Ernout, A., Meillet, A., 19594, Dictionnaire étymologique de la langue latine. Histoire des mots, Paris [1932¹].

Етснето, H., 2012, Les Scipions. Famille et pouvoir à Rome à l'époque républicaine, Bordeaux.

Fezzi, L., 2013, Catilina. La guerra dentro Roma, Napoli.

GaвbA, E., 1951, «L'esercito professionale da Mario ad Augusto», Athenaeum 29, p. 171-272.

GabBA, E., 1956, «Il ceto equestre e il senato di Silla», Athenaeum 34, p. 124-138.

GaBBA, E., 1973, Esercito e società nella tarda repubblica romana, Firenze.

GelZer, M., 1923, «Sergius», 23, in RE, II.A.2, c. 1693-1711.

Girardet, K. M., 2001, «Imperia und provinciae des Pompeius 82 bis 48 v. Chr.», Chiron 31, p. 153-209.

Hantos, T., 1980, Res publica constituta. Die Verfassung des Dictators Sulla, Stuttgart.

Harmand, J., 1967, L'armée et le soldat à Rome de 107 à 50 avant notre ère, Paris.

Havas, L., 1974, «Catilina en Hispanie ultérieure?», WZRostock 23, p. 229-232.

HAwTHORN, J.R., 1962, «The senate after Sulla», GerR 9, p. 53-60. 
Heftner, H., 1994, Plutarch und der Aufstieg des Pompeius. Ein historischer Kommentar zu Plutarchs Pompeiusvita, Teil I: Kap. 1-45, Frankfurt am Main.

HiLl, H., 1932, «Sulla's new senators in 81 B.C.», CQ 20, p. 170-177.

Hillman, T.P., 1998, «Pompeius' imperium in the war with Lepidus», Klio 80, p. 91-110.

HinARD, F., 1984, «La male mort. Exécutions et statut du corps au moment de la première proscription», in Du châtiment dans la cité. Supplices corporels et peine de mort dans le monde antique. Table ronde (Rome, 9-11 novembre 1982), Paris - Roma, p. 295-311.

Hinard, F., 1985, Sylla, Paris.

Hinard, F., 1986, «Mais qui a donc tué Gratidianus?», Kentron 2, p. 118-122.

Hinard, F., 2011, Rome, la dernière République. Recueil d'articles, Bordeaux.

Hoffmann, W., 1959, "Catilina und die Römische Revolution», Gymnasium 66, p. 459-477.

Holmes, T.R., 1923, The Roman republic and the founder of the empire, I, Oxford.

HÜLSEN, C., 1894a, «Aesernia», in RE, I, c. 684-685.

HülsEn, C., 1894b, «Alba Fucens», in RE, I, c. 1300-1301.

JAL, P., 1984, Abrégés des livres de l'Histoire romaine de Tite-Live, I, Paris.

Kajanto, I., 1965, The Latin cognomina, Helsinki.

Keaveney, A., 1982¹, Sulla. The last republican, London - Canberra.

Keaveney, A., 2005a², Sulla. The last republican, London - New York.

Keaveney, A., 2005b, «The terminal date of Sulla's dictatorship», Athenaeum 93, p. 423-439.

Keaveney, A., Strachan, J. C. G., 1981, «L. Catilina legatus: Sallust, Histories I. 46M», CQ 31, p. 363-366.

KLEBS, E., 1894, «Aemilius», 81, in RE, I, c. 564-565.

Kromayer, J., Veith, G., 1928, Heerwesen und Kriegführung der Griechen und Römer, München.

La Penna, A., 1963, «Per la ricostruzione delle Historiae di Sallustio», SIFC 35, p. 5-68.

La Penna, A., Funari, R., 2015, C. Sallusti Crispi Historiae, I, Berlin - Boston.

LABRUnA, L., 1975, Il console sovversivo: Marco Emilio Lepido e la sua rivolta, Napoli.

Levick, B., 2015, Catiline, London - New York.

LEwIs, R.G., 2001, «Catilina and the Vestal», CQ 51, p. 141-149.

Manni, E., 1969², Lucio Sergio Catilina, Palermo [1939¹].

Marshall, B., 1985, "Catilina and the execution of M. Marius Gratidianus», CQ 35, p. 124-133.

Maurenbrecher, B., 1893, C. Sallusti Crispi Historiarum reliquiae, II, Leipzig.

Mazzarino, S., 1966, Il pensiero storico classico, II.1, Bari.

McGushin, P., 1977, C. Sallustius Crispus. Bellum Catilinae. A commentary, Leiden.

McGushin, P., 1992, Sallust. The histories, I, Oxford.

Nicolet, C., 1966, L'ordre équestre à l'époque républicaine (312-43 av. J.-C.), I, Paris.

Niedermann, M., 1935, «Mélanges de linguistique latine», Mnemosyne 3, p. 265-278.

Oтто, W., 1907-1913, «Catilina», TLL - Onomasticon, II, c. 259-261.

PAreti, L., 1965, «Catilina», in Studi minori di storia antica, III, Roma, p. 291-444 [= La congiura di Catilina, Catania, 1934].

Premerstein, A. von, 1924, «Legatus», in RE, XII.1, c. 1133-1149.

Ramsey, J. T., 2007², Sallust's Bellum Catilinae, Oxford [1984ㄹ․

RAMSEY, J.T., 2019, «The date of the consular elections in 63 and the inception of Catiline's conspiracy», HSPh 110 , in corso di stampa.

RAwson, E., 1987, «Sallust on the eighties?», CQ 37, p. 163-180.

SANTANGelo, F., 2006, «Sulla and the Senate: a reconsideration», CCG 17, p. 7-22.

Santangelo, F., 2014, «Sempre poco allineati: il decennio dopo Silla», in R. Cristofoli, A. Galimberti, F. Rohr Vio (ed.), Lo spazio del non-allineamento a Roma fra tarda repubblica e primo principato. Forme e figure dell'opposizione politica. Atti del convegno di studi (Milano, 11-12 aprile 2013), Roma, p. 1-23. 
Schietinger, G.-P., 2017, «Lucius Sergius Catilina. Karriereperspektiven und Karriere eines homo paene novus in der späten Römischen Republik», Klio 99, p. 149-191.

Schleussner, B., 1978, Die Legaten der römischen Republik. Decem legati und ständige Hilfsgesandte, München.

SмIth, R.E., 1958, Service in the post-marian Roman army, Manchester.

SteEL, C., 2014a, «Rethinking Sulla: the case of the Roman senate», CQ 64, p. 657-668.

SteEL, C., 2014b, «The Roman senate and the post-Sullan res publica», Historia 63, p. 323-339.

SuOLAhti, J., 1955, The junior officers of the Roman army in the republican period, Helsinki.

Syme, R., 1964, Sallust, Berkeley - Los Angeles.

TAYLOR, L. R., 1942, «Caesar's colleagues in the pontifical college», AJPh 63, p. 385-412.

Thомаsson, B.E., 1991, Legatus. Beiträge zur römischen Verwaltungsgeschichte, Stockholm.

Ungern-Sternberg, J. von, 1997, «Das Verfahren gegen die Catilinarier, oder: Der vermiedene Prozeß», in U. Manthe, J. von Ungern-Sternberg (ed.), Große Prozesse der römischen Antike, München, p. 85-99, 204-206.

URso, G., (c.d.s.), Catilina. Le faux populiste, Bordeaux.

VERVAet, F., 2009, «Pompeius' career from 79 to 70 BCE: constitutional, political and historical considerations», Klio 91, p. 406-434.

Walde, A., Hofmann, J.B., 19825 Lateinisches etymologisches Wörterbuch, I, Heidelberg [1938; $\left.1906^{1}\right]$.

Willems, P., 1878, Le sénat de la République romaine. Sa composition et ses attributions, I, Louvain.

WöLfFlın, E., 1884, «Catilinarius», Archiv für lateinische Lexikographie 1, p. 277-279. 